\title{
Le congrès des élus départementaux et régionaux et l'ambiguité des revendications statutaires
}

La « dignité » comme projet - débat statutaire et limites de la politique locale à la Martinique

The Congress of general and regional councillors and the ambiguity of status claims: the "dignity" as project - the status debate and the limit of local politics in Martinique.

Ulrike Zander

\section{(2) OpenEdition}

Journals

Édition électronique

URL : http://journals.openedition.org/plc/147

DOI : $10.4000 /$ plc. 147

ISSN : 2117-5209

Éditeur

L'Harmattan

\section{Édition imprimée}

Date de publication : 15 décembre 2007

Pagination : 15-39

ISBN : 2-296-01986-2

ISSN : 1279-8657

Référence électronique

Ulrike Zander, « Le congrès des élus départementaux et régionaux et l'ambiguïté des revendications statutaires », Pouvoirs dans la Caraibe [En ligne], 15 | 2007, mis en ligne le 05 mai 2011, consulté le 10 décembre 2020. URL : http://journals.openedition.org/plc/147 ; DOI : https://doi.org/10.4000/plc. 147 


\title{
LE CONGRES DES ELUS DEPARTEMENTAUX ET REGIONAUX ET L'AMBIGUITE DES REVENDICATIONS STATUTAIRES :

\author{
LA « DIGNITE » COMME PROJET - DEBAT STATUTAIRE ET LIMITES \\ DE LA POLITIQUE LOCALE A LA MARTINIQUE
}

\author{
Ulrike ZANDER \\ Doctorante à l'Ecole des Hautes Etudes en \\ Sciences Sociales, Paris \\ et à la Freie Universität Berlin \\ Membre du CRPLC \\ Université des Antilles et de la Guyane
}

Depuis l'abolition de l'esclavage en 1848, l'histoire politique de la Martinique a été fortement marquée par la question du statut, qui interroge sans cesse le lien avec la France dans le but de le redéfinir. Elle domine le débat politique local et détermine les clivages. En effet, le paysage politique martiniquais est structuré par les revendications portant sur le statut (Daniel, 2002 : 589). Si pendant un siècle (de 1848 à 1946) le débat statutaire fut fondé sur la revendication assimilationniste ${ }^{1}$, il a viré dans les années qui suivirent la départementalisation totalement à l'opposé, donnant lieu à des revendications nationalistes : autonomistes comme indépendantistes. Dans l'esprit des instigateurs de la départementalisation, principalement les députés Césaire et Bissol (communistes) de la Martinique, Monnerville (radical-socialiste) de la Guyane et Vergès (communiste) de la Réunion, l'assimilation des «quatre vieilles » colonies $^{2}$ de la France au système législatif en vigueur en métropole avait pour objectif d'établir l'égalité entre les peuples d'outre-mer et celui de la métropole ${ }^{3}$ et d'améliorer les conditions de vie de la population.

La loi de départementalisation du 19 mars 1946 fut adoptée à l'unanimité. Par la suite, il apparut clairement que la réticence des autorités nationales contrasta avec l'enthousiasme affiché des populations locales (Rubio, 2000: 26), et quelques années plus tard seulement, ce fut le désenchantement du coté des Martiniquais, la réalité de la départementalisation ne correspondant pas à leurs aspirations. Ce furent alors

\footnotetext{
${ }^{1}$ Cf. William (1981 : 50-61).

${ }^{2}$ La Guadeloupe, la Guyane, la Martinique et La Réunion sont aussi appelées les «quatre vieilles » colonies à cause de leur colonisation précoce par rapport aux colonies plus récentes.

${ }^{3}$ Gaston Monnerville, Après la fraternité et la liberté, nous venons demander l'égalité devant la loi, l'égalité des droits, Assemblée nationale constituante, $1^{\text {ère }}$ séance du 14 mars 1946, JORF débats parlementaires 1946, p.755, cité par Rubio, $2000: 27$.
} 
ses initiateurs qui contestèrent les premiers l'idéologie assimilationniste et lancèrent le débat statutaire. En effet, «la départementalisation joue comme révélateur de la persistance d'une situation coloniale » (Suvélor, 1983 : 2202) où « à la fatalité biologique de l'esclavage a succédé la fatalité économique, au complexe d'infériorité le complexe de la dépendance inéluctable, mais vécu dans le ressentiment » (idem : 2199).

C'est effectivement à partir du constat d'échec de la départementalisation que la revendication nationaliste prit son essor. L'affirmation identitaire martiniquaise est ainsi en quelque sorte « un fruit de la départementalisation » (Michalon, $2002: 12$ ).

Nous n'allons pas présenter tous les détails historiques du débat statutaire, ceci n'est pas l'objet de cet article. Cependant, pour terminer ce bref propos descriptif, on peut constater que 60 ans se sont écoulés depuis la départementalisation - événement qui constitue sans doute, avec l'abolition de l'esclavage, le fait le plus marquant de l'histoire martiniquaise - et la question du statut semble rester enfermée dans un éternel cercle vicieux.

Certes, à l'arrivée des socialistes au pouvoir en France en 1981, l'État français entama des réformes institutionnelles importantes, en particulier avec la première décentralisation (loi du 2 mars 1982). Depuis, les collectivités départementale et régionale sont superposées et disposent de compétences élargies. Cependant, selon les termes de Justin Daniel, la décentralisation de 1982 fut plutôt un "ajustement à la marge » qu'un réel changement, et renforça même le mode de régulation socio-politique inauguré avec la départementalisation (Daniel, 1989).

Le dernier épisode - jusqu'ici - du débat statutaire à rebondissements débuta en 1998, quand le gouvernement Jospin lança sa réflexion sur l'avenir institutionnel des DOM, laquelle aboutit au rapport Lise-Tamaya et à la loi d'orientation pour l'outre-mer. Ces derniers textes furent d'ailleurs contestés par les trois présidents des régions Guadeloupe, Guyane et Martinique, respectivement Lucette Michaud-Chevry, Antoine Karam et Alfred MarieJeanne, lesquels signèrent la Déclaration de Basse-Terre du $1^{\text {er }}$ décembre 1999, véritable manifeste pour l'autonomie. En Martinique, les deux évènements fondamentaux de cette période furent le Congrès des élus départementaux et régionaux ainsi que la consultation populaire du 7 décembre 2003, laquelle clôtura cet épisode. Il est vrai que certains 
Martiniquais espéraient régler définitivement la question du statut par cette consultation. Mais comme l'actualité nous le montre, cela fut une illusion, car cette question continue à occuper les premiers rangs de la scène politique et à déterminer les clivages. Ainsi, de nombreux élus souhaitent déjà organiser une nouvelle consultation sur la question des institutions.

En tant qu'observatrice extérieure, nous sommes alors amenée à nous poser la question de savoir pourquoi le problème du statut de la Martinique est si difficile, voire impossible à résoudre... Nous n'avons pas la prétention d'apporter une réponse exhaustive à cette interrogation car nous ne disposons pas des connaissances politistes et juridiques nécessaires pour le faire. Cependant, nous tentons d'indiquer quelques pistes de réflexion fondées sur l'étude d'un événement considéré par certains comme historique: la deuxième session du Congrès des élus départementaux et régionaux laquelle s'est tenue en février/mars $2002^{4}$. Nous tenons à préciser qu'il ne s'agit pas de l'étude de cet événement en soi mais d'une démarche anthropologique qui met l'accent sur les réactions de la population martiniquaise à ce Congrès et les représentations qu'elle en a. Quand nous employons ici le terme "population », nous désignons les individus qui ne font pas partie de l'élite intellectuelle et politique. Nous avons en effet choisi une démarche centrée sur l'étude des représentations populaires de l'identité et en particulier de l'identité nationale, car ce domaine a été très peu exploré jusqu'à présent en Martinique, alors que beaucoup de travaux ont déjà été menés sur la conscience identitaire et nationale de l'élite intellectuelle et politique, en particulier par les intellectuels martiniquais eux-mêmes.

Le matériau empirique a été constitué à partir de 28 entretiens semidirectifs enregistrés ${ }^{5}$, de nombreuses discussions informelles non-enregistrées

\footnotetext{
${ }^{4}$ Le président du Conseil régional Alfred Marie-Jeanne notamment qualifie le vote pour la nouvelle collectivité territoriale d'historique (Procès-verbal in-extenso, tome 1, 20 février 2002 : 92). Le conseiller général Garcin Malsa déclare qu' "aujourd'hui nous sommes à un carrefour historique de notre histoire » (PV, 2002, tome 1, p.109. L'hebdomadaire Antilla du $1^{\text {er }}$ mars 2002 intitule son dossier traitant du Congrès : "Congrès : L'Histoire en marche ». Cependant, certains observateurs sont beaucoup plus prudents, par exemple, Tony Delsham qui commence son article du même hebdomadaire avec cette phrase: "La suite des événements nous dira si, en ce mois de février 2002, les Martiniquais ont effectivement participé à la rédaction d'une page de leur Histoire ou si, au contraire, nous étions en plein délire tropical sous l'œil ricanant de Paris (...)» (Antilla, $\mathrm{n}^{\circ}$ 977, p.13).

${ }^{5}$ Pour une description de l'échantillon voir annexe p. 20 et 21.
} 
ainsi que de l'observation participante. Il s'agit d'une enquête qualitative qui ne peut effectivement fournir des données représentatives de la population martiniquaise.

La réunion du Congrès avait été prévue par le gouvernement Jospin dans la «Loi d'orientation pour l'outre-mer» du 13 décembre 2000 comme cadre d'expression offert aux élus locaux de chaque DOM/ROM afin qu'ils fassent part de leurs propositions concernant l'évolution institutionnelle de leur département. Le Congrès devait réunir l'ensemble des élus appartenant au Conseil général et au Conseil régional ainsi que les députés. Même si les propositions du Congrès devaient avoir un caractère strictement indicatif - le gouvernement étant libre d'y donner suite ou pas - le processus engagé fut nouveau. Pour la première fois, le gouvernement français faisait, dans une certaine mesure, un pas vers les élus locaux en leur proposant d'élaborer eux-mêmes des suggestions sur l'avenir institutionnel de chacun des DOM/ROM. De ce point de vue, le Congrès peut effectivement être qualifié d'historique.

En Martinique, le Congrès connut trois sessions : la première le 12 juin 2001, la deuxième les 20,21 et 23 février ainsi que le 4 mars 2002, et la troisième le 10 avril 2003. Dans notre enquête, nous considérons essentiellement la deuxième session car il nous semble qu'elle fut la plus importante, puisqu'elle aboutit à un certain nombre de propositions concrètes ${ }^{6}$. Tout d'abord, après de longs débats acharnés, la grande majorité des élus (62 sur 84 ) - toutes tendances politiques confondues - se prononça pour la sortie du statut départemental et la création d'une nouvelle collectivité territoriale de la Martinique. Dans cette nouvelle collectivité territoriale, les deux assemblées locales (Conseil général et Conseil régional) devaient être remplacées par une assemblée unique élue au scrutin proportionnel dans le cadre d'une circonscription unique avec un seuil de 5\% des suffrages exprimés. Pour cette nouvelle assemblée, la majorité des élus réclamèrent des compétences élargies, un pouvoir réglementaire et un pouvoir législatif « encadré » ${ }^{7}$.

\footnotetext{
${ }^{6}$ Les débats de la deuxième séance du Congrès sont retranscrits dans leur intégralité dans le

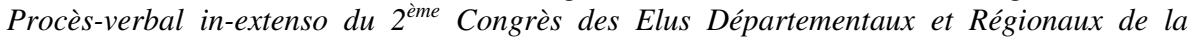
Martinique, 4 tomes, mars 2002. Pour un résumé de cet événement voir la presse locale, notamment le quotidien France-Antilles et l'hebdomadaire Antilla, n 977 et 978.

${ }^{7}$ Pour plus de détails cf. Antilla, ${ }^{\circ}$ 978, p.3-6.
} 
A coté de ces revendications concrètes, les élus locaux se livrèrent à de longues polémiques sur la question identitaire, très mouvementées et hautement théoriques, initiées par un amendement proposé par le conseiller général et militant indépendantiste Francis Carole revendiquant la reconnaissance d'une nation martiniquaise et d'un peuple martiniquais ${ }^{8}$.

\section{Manque d'adhésion, désintérêt, mauvaise compréhension...}

Comment la population martiniquaise a-t-elle réagi à cet événement politique particulier qu'était le Congrès ? Premier constat: un certain nombre de personnes figurant dans notre échantillon avaient l'impression d'assister à un spectacle comique plutôt qu'à une réunion politique sérieuse ayant pour enjeu l'avenir de la Martinique :

« Je n'avais pas l'impression d'assister à un Congrès. Ca me rappelle mes classes, quand j'étais au collège, à la salle de permanence, où on chahutait et tout le monde disait : taisez-vous, taisez-vous. (...) C'est une honte. Alors, bon, je ne dis pas que le résultat soit une honte, mais on ne peut pas se dire être politique, représenter autant de personnes, et débattre au Congrès en tout ça de temps, avec autant de polémiques. (...) Ca m'a dégoûté. » (Une femme de 32 ans, résidant à Rivière-Pilote et travaillant dans le milieu associatif).

«Franchement, c'était un cancan, ils se comportaient comme des enfants de la maternelle, vraiment. Parce que normalement, ils étaient là pour travailler, mais... ils se tiraient l'un sur l'autre, alors je dis que si c'est comme ça, la Martinique ne va pas avancer. » (Une femme, 33 ans, Sainte-Anne, emploi-jeunes).

A cette sensation de ridicule s'ajoutait la conviction que de toutes les façons, quel que fût l'enjeu des débats, l'objectif réel des élus n'était pas de faire avancer «le pays», mais de servir leurs ambitions personnelles en termes de carrière politique :

« (...) les gens se battent et ils ne savent pas pourquoi. Ils ont mis un Congrès, celui-là va dire que c'est lui qui veut être à la tête, mais ils vont se battre pour savoir qui sera à la tête, qui ne sera pas, et ça ne va pas avancer (...)» (Propos d'une femme d'une quarantaine d'années, résidant à Sainte-Anne, sans profession).

${ }^{8}$ Cf. PV, 2002, tome $1: 12$. 
$\mathrm{Au}$ vu de ces premières réactions, on constate un réel manque d'adhésion à ce processus politique que constituait le Congrès. En général, les personnes que nous avons pu interroger n'y croyaient pas, non seulement parce qu'elles trouvaient les élus ridicules et les soupçonnaient d'intentions purement politiciennes, mais aussi parce qu'elles ne se faisaient guère d'illusions quant à l'impact de cet événement au niveau national.

«(...) les solutions ne sont pas prises ici. Tout ce qu'on demande et tout, mais rien n'est vraiment pris en compte. » (Un homme, 27 ans, chômeur, Sainte-Anne).

« Ce sera toujours la France qui aura le dernier mot. Même si elle accorde des compétences aux politiques martiniquais. Les Français vont boucher les yeux aux politiques. » (Un homme, 31 ans, ouvrier dans le bâtiment, Rivière-Pilote).

Cette désillusion engendrait par ailleurs en réel désintérêt. Nous avons rencontré de nombreuses personnes affirmant que les débats au Congrès ne les intéressaient pas du tout, qu'elles ne les avaient pas suivis dans les médias. Parmi ceux qui avaient fait l'effort de les suivre, nous avons pu en observer qui s'endormaient devant leurs écrans de télévision. Il faut dire, à leur décharge, que le débat le plus théorique, le plus complexe et peut-être aussi le plus soporifique pour certains (le peuple et la nation, nous allons y revenir) se tenait à une heure avancée de la soirée.

On constate par ailleurs un manque de compréhension au niveau général des propositions du Congrès. Très peu de personnes de notre échantillon avaient saisi tous les éléments concernant les motions et amendements votés. Les débats étaient trop techniques pour être compréhensibles par une majorité de la population. Visiblement, les élus locaux n'ont pas su se mettre à la portée de leurs administrés pour leur faire comprendre les tenants et aboutissants de cette réunion particulière que constituait le Congrès. Le même phénomène a pu être constaté plus tard, lors de la campagne électorale pour la consultation du 7 décembre. Ce défaut de communication des élus envers la population est un facteur substantiel parmi l'ensemble des facteurs qui créent un réel décalage entre la population et ses élus. 
Une conception ethno-culturelle de la nation...

Nous allons maintenant nous intéresser à un aspect spécifique de ce décalage en examinant de plus près l'amendement Carole sur la reconnaissance de la nation martiniquaise et du peuple martiniquais. Francis Carole expose les fondements de sa revendication comme suit :

«(...) Nous constituons, en effet, un peuple c'est-à-dire une communauté d'hommes fondée sur des apports divers, d'Afrique, d'Europe, d'Asie, sur un territoire clairement identifié. Nous avons une culture qui n'est pas la culture française. Nous avons des mours, une langue, un imaginaire qui ne sont pas les mêmes qu'en France. Nous avons aussi ce sentiment d'appartenance à un groupe, à une communauté. C'est ce qui fonde l'existence d'une nation martiniquaise ; c'est ce qui fonde l'existence d'un peuple martiniquais. ${ }^{9}(\ldots)$ tout le débat politique du demi-siècle qui vient de s'écouler est un débat autour de la reconnaissance du peuple martiniquais et de son droit à l'autodétermination. (...) à terme notre peuple a vocation à se constituer en Etat souverain (...)» (Procèsverbal in extenso du $2^{e}$ Congrès de Elus Départementaux et Régionaux de la Martinique, 2002, tome 1 : 12).

Dans ces propos, Francis Carole indique clairement que, pour lui, les deux notions «peuple» et «nation» sont indissociables. Il définit cet ensemble «peuple-nation » par l'existence d'une communauté vivant sur un territoire, dotée d'une culture et dont les membres ont le sentiment d'appartenir à cette communauté. Son collègue Louis Boutrin ${ }^{10}$ va dans le même sens, mais en précisant par ailleurs que la notion de nation, tout en étant indissociable de celle de peuple n'est pas liée à celle d'Etat :

« (...) La nation martiniquaise existe et je dois dire aujourd'hui que même si nous ne constituons pas un Etat, aujourd'hui le peuple martiniquais vit dans la nation martiniquaise. (...) la reconnaissance du peuple martiniquais veut dire que l'on reconnaît l'existence de la nation martiniquaise. (...)» (PV, 2002, tome 1 : 93).

\footnotetext{
${ }^{9}$ C'est nous qui soulignons.

${ }^{10}$ Conseiller régional appartenant au mouvement «Bâtir le Pays Martinique ».
} 
Daniel Marie-Sainte ${ }^{11}$ ajoute aux caractéristiques définissant cet ensemble peuple-nation celle d'une histoire commune :

«(...) Existe-t-il des peuples sans nation ? Or, notre peuple, il y a quelque chose qui le caractérise, c'est qu'il a un territoire bien défini. (...) et même lorsque ses individus sont natifs de l'autre rive de l'Atlantique, continuent à se considérer comme appartenant à ce pays. C'est ce qui fonde la nation. Nous avons une histoire commune, une culture commune, le sentiment d'appartenir à une communauté. (...)» (PV, 2002, tome $1: 93)$.

Il mentionne plus loin son désir de voir la France évoluer en «Etat multinational » :

«(...) Je rêve d'un jour où les différents peuples qui composent la France vont poser cette revendication tellement fort que le Parlement, la constitution vont finir par reconnaître que la France est un Etat multinational. (...). » (PV, 2002, tome 1 : 101).

Pour Daniel Marie-Sainte, il existe donc le peuple martiniquais qui forme la nation martiniquaise, une nation qui fait partie d'un ensemble de nations doté d'un Etat qui est la France.

Avant de consacrer quelques réflexions à l'idée de nation avancée par les élus cités ci-dessus, pour ensuite la mettre en relation avec les représentations de la population martiniquaise telles qu'elles se dégagent de notre matériau empirique, il nous semble important au préalable, d'ouvrir une parenthèse sur le concept de la nation, sans pour autant entrer dans les méandres du débat scientifique autour de ce concept si controversé. En réalité, il n'existe pas de définition claire et universellement admise de la nation ${ }^{12}$. Cependant, beaucoup d'auteurs retiennent comme caractéristique centrale d'une nation le fait que ses membres manifestent le sentiment d'y appartenir. Ainsi, pour Hugh Seton-Watson, "tout ce que je peux trouver à dire c'est qu'une nation existe quand un nombre significatif de gens dans une communauté se considèrent comme formant une nation, ou se conduisent

${ }^{11}$ Conseiller régional appartenant au MIM (Mouvement Indépendantiste Martiniquais).

12 Comme l'a noté Harold R. Isaacs, "la 'nation' - si formidablement réelle dans le monde réel de tout un chacun au quotidien - a échappé à tous les efforts des savants pour s'accorder sur ce qu'elle signifie exactement »(Isaacs, 1975 : 174, notre traduction). 
comme s'ils en formaient une "(Seton-Watson, 1977: 5, notre traduction). Eric Hobsbawm quant à lui pose l'hypothèse de travail suivante: "tout groupe suffisamment important en nombre dont les membres se considèrent comme faisant partie d'une même nation sera considéré comme tel » (Hobsbawm, 1990). Pour Georg Elwert, la nation "n'exige pas de caractéristiques culturelles communes, cependant elle implique que la majorité de ses membres croie qu'un certain nombre d'éléments communs les rassemble tous dans une seule structure sociale» (Elwert, 2001 : 252, notre traduction).

Sans multiplier les exemples, nous retenons que la définition de la nation, sa représentation, doit émaner de l'intérieur de chaque société concernée. De ce point de vue, la polémique engendrée par l'amendement Carole peut être considérée comme un cas exemplaire du débat «national » martiniquais.

Dans l'idée de la nation défendue par Francis Carole et d'autres élus du camp nationaliste, la caractéristique la plus fondamentale d'une nation est la culture que partage une communauté d'individus sur un même territoire. En d'autres mots, la construction de la nation martiniquaise est fondée avant tout sur une culture martiniquaise pratiquée par un peuple martiniquais, une culture différente d'autres cultures et notamment de la française. Non seulement en Martinique, mais partout dans le monde, l'argument d'une particularité culturelle est souvent mis en avant pour justifier une affirmation nationaliste ${ }^{13}$. Il s'agirait alors d'une conception ethno-culturelle, à l'instar de la conception «allemande » de la nation établie à la fin du $18^{\mathrm{e}}$ siècle par le philosophe Herder, selon lequel chaque nation est le résultat d'une culture propre qui se transmet dans le temps, une sorte d'héritage s'imposant, par exemple, à travers une langue maternelle ${ }^{14}$. Cette conception pose néanmoins quelques

\footnotetext{
13 «Le présupposé par lequel on prétend le plus souvent légitimer une affirmation nationaliste postule que ce serait l'unité culturelle du "peuple», son identité comme on aime à le dire aujourd'hui, qui fonderait le principe et l'existence même de la nation en tant qu'identité politique. Un présupposé qui implique donc qu'il y aurait des sociétés monoculturelles. Or de telles sociétés n'existent pas en fait. (...) (Giraud, $2000: 88$ ).

${ }^{14}$ On oppose souvent cette conception «allemande » de la nation ethnique ou nation-génie à celle présentée par Ernest Renan en 1882 et appelée «française» de la nation civique ou nation-contrat. Selon Renan, la nation ne dépend ni d'une race particulière, car « au principe des nations on substitue alors celui de l'ethnographie », ni d'une langue, ni d'une religion, ni d'intérêts économiques partagés, ni encore de la géographie; la nation est une âme, un principe spirituel... l'existence d'une nation est un plébiscite de tous les jours ».
} 
problèmes concrets: si la Martinique est donc une nation fondée sur le particularisme culturel du peuple martiniquais, comment peut-on définir la nationalité martiniquaise ? En effet, déjà la notion de peuple soulève un certain nombre de questions fondamentales : quelles sont les caractéristiques propres à un individu définissant son appartenance à un peuple donné, et non pas à un autre ? Son lieu de naissance, ses origines, sa pratique culturelle, son amour pour tel ou tel « pays »... ? Et comment classer les individus d'origine mixte ? En réalité, le peuple comme donnée essentielle et homogène, revendiquée par les nationalistes, n'existe pas. D'après le «droit du sang», inhérente à la conception ethno-culturelle, seraient inclus dans cette construction de «peuple-nation» tous les Martiniquais de la diaspora, vivant en France métropolitaine ou ailleurs, même de deuxième et troisième génération. Et pourtant, certains d'entre eux n'ont jamais foulé le sol martiniquais, beaucoup ne parlent pas la langue maternelle qui est le créole et ne sont vraisemblablement plus des porteurs de cette culture martiniquaise à laquelle les nationalistes martiniquais font référence. D'un autre côté, quel serait le sort de toute une communauté étrangère vivant sur le sol martiniquais, Haïtiens et Sainte-Luciens en majorité (ne parlons pas des «Métropolitains », car ceci est encore un autre problème $)^{15}$ ?

En réalité, la culture est «idéologisée » et dans une certaine mesure inventée. Car cette "espèce de tradition qu'on essaie désespérément de revaloriser et faire revivre, a bien sûr un contenu différent et une fonction politique différente de celle des générations précédentes, lesquelles n'ont jamais objectivisé leur culture comme quelque chose qui ne serait pas une partie d'eux-mêmes»(Eriksen, 1992: 10, notre traduction). Ainsi, Michel Giraud parle d'une "relation d'extériorité » de la population «face à ce qui est proclamé être leur culture» (Giraud, 1999: 380). Si la culture est inventée, les frontières culturelles aussi relèvent d'une construction (Giraud, 2000: 88), une construction obéissant à des stratégies bien conscientes "dans une lutte pour le pouvoir s'organisant autour de programmes économiques, politiques et sociaux plus ou moins conditionnés par la question du statut »(Daniel, $\left.2002: 595^{16}\right)$.

\footnotetext{
${ }^{15}$ Edouard Glissant en se référant à l'appel au lynchage des Haïtiens en Guadeloupe par Ibo Simon en février 2002 parle de «la difficulté à concevoir l'idée de nation, et à l'inverse les perversions de cette idée quand elle s'exaspère en pure xénophobie » (in Antilla n 977, p. 23).

${ }^{16}$ Justin Daniel parle d'ailleurs d'une « politisation des identités ».
} 


\section{... versus une conception politico-économique}

Au-delà de la conception de la nation défendue par les élus incarnant la mouvance nationaliste, quelles sont les représentations au sein de la population martiniquaise de la nation et du peuple ? La nation martiniquaise et le peuple martiniquais existent-ils pour ces personnes que nous avons pu rencontrer?

Tout d'abord, en ce qui concerne la notion de «peuple martiniquais », il ressort de l'examen de l'échantillon que l'idée du «peuple martiniquais » se trouve bien enracinée dans les consciences. Les enquêtés ont le sentiment d'appartenir à un peuple spécifique qui se différencie d'autres peuples dont le peuple français. Et ce peuple est défini à l'instar des nationalistes, en termes culturalistes : il existe un peuple martiniquais parce qu'il existe une culture martiniquaise, une langue créole, des mœurs et des habitudes bien spécifiques, une façon d'être particulière etc. Par contre, pour nos interlocuteurs, l'indissociabilité entre le peuple et la nation, soulignée et considérée comme fait irréfutable par les nationalistes, est tout sauf évidente. $\mathrm{Au}$ contraire, ce sont pour eux deux choses bien distinctes et tout amalgame supposerait un danger (d'indépendance). Certes, nous avons pu noter de rares exceptions où les personnes rejoignaient la conception ethno-culturelle des nationalistes, notamment parce qu'elles font elles-mêmes partie de la mouvance nationaliste ou en sont proches. Cependant, selon une large majorité des personnes rencontrées, ce qui caractérise une nation, ce sont essentiellement ses dimensions politique et économique, alors que ces dimensions font totalement défaut dans la conception soutenue par les nationalistes. Pour exister en tant que nation, deux conditions sont en général mises en avant: l'auto-gestion (l'indépendance politique) et l'autosuffisance (l'indépendance économique) :

«Savoir se gérer, avoir tout ce qu'il faut dans son pays pour savoir se gérer. Si jamais tu veux avoir ton drapeau, il faut savoir te gérer, il faut qu'il y ait une ouverture (...) » (Un homme, 35 ans, employé de la mairie, Sainte-Anne).

«Une nation, c'est subvenir à ses propres besoins, produire et pouvoir vendre, pouvoir acheter avec l'argent du pays, et puis avoir des gens bien placés, un président qui n'ait pas la grosse tête, un gars qui comprenne un petit peu les gens c'est tout. Et puis, s'entraider, essayer d'aider les plus démunis. (...) » (Homme, 27 ans, chômeur, Sainte-Anne). 
Cet homme évoque aussi la solidarité nationale qui présuppose l'adhésion aux principes de la nation. Par ailleurs, pour mes interlocuteurs, à l'existence d'une nation est obligatoirement liée celle d'un Etat. Ainsi, « nation martiniquaise » signifie «indépendance », et l'indépendance est la condition nécessaire et préalable à l'avènement de la nation et non l'inverse ${ }^{17}$, comme l'illustrent ces propos :

«(..) pour prétendre que vous êtes une nation, il faut que vous soyez indépendant (...)» (un homme de 63 ans, militaire à la retraite, Schoelcher).

« Pour qu'il y ait une nation, il faut qu'il y ait un Etat. Pour qu'il y ait un Etat, il faut que la France nous donne cet Etat, nous laisse avoir cet Etat. Il faut que nous soyons indépendants, c'est clair. On ne peut pas parler d'une nation, si on parle seulement d'une nation culturelle (...). Quand on parle de nation, tout le monde voit indépendance. (...). (En parlant de l'amendement Carole) : C'est une manière très comique, très vicieuse, très perverse et dangereuse de dire que la Martinique est indépendante. (...)» (Une femme de 32 ans, travaillant dans le milieu associatif, Rivière-Pilote).

L'idée selon laquelle l'indépendance doit nécessairement précéder la nation prend le contre-pied du projet nationaliste. Car c'est souvent l'orientation vers un Etat désiré et en attente de réalisation qui caractérise un tel projet (Roger, $2001: 2$; Elwert, $2001: 252$ ). La conception populaire de la nation qui se dégage de notre matériau empirique peut ainsi être qualifiée d'anti-nationaliste. Le terme "nation » évoque pour certains même une « vision d'horreur » impliquant le « largage », la perte des «droits acquis », voire la dictature...

Nous nous trouvons alors face à un réel hiatus entre la conception «populaire» de la nation et celle des nationalistes martiniquais, un hiatus dont ces derniers sont d'ailleurs bien conscients. Lors du Congrès, Camille

\footnotetext{
${ }^{17}$ Selon Roland Suvélor, «on peut faire une classification en trois étapes : il y a d'abord le peuple, un ensemble d'individus. Le peuple devient une nation dans la mesure où il y a une cohésion, communauté de l'homme, de sentiments, de pensées, de projets. Donc, le peuple forme une nation. Et finalement la nation devient un Etat, l'Etat étant si vous voulez la marque de reconnaissance, disons extérieure de l'existence d'une nation. » (Entretien du 18 février 2002).
} 
Darsières, élu du PPM et nationaliste de la première heure, auteur de l'ouvrage fréquemment cité "De l'origine de la nation martiniquaise » (1974), l'a même avancé comme raison de ne pas participer au vote sur la reconnaissance de la nation martiniquaise :

«(...) j'ai peur aujourd'hui que nous ne fassions, malgré nous, un recul, que nous fassions au niveau du peuple martiniquais une rétraction (...). J'ai peur qu'ils ne joignent les deux idées d'une nouvelle collectivité qui est une avancée institutionnelle et puis d'une notion de nation que le peuple ne connaît pas ${ }^{18}$. N'allez pas compter sur nos juristes, sur nos constitutionalistes, sur nos publicistes pour apprendre ce qu'est la nation martiniquaise. Je ne leur en tiens pas rigueur ; ils n'apprendront pas la nation parce qu'ils ne l'ont pas apprise eux-mêmes et que pour être un juriste avancé, il faut être un juriste engagé. Alors nous sommes dans la situation suivante: ma conviction que je suis une partie de la nation martiniquaise et ma peur que le peuple martiniquais en entendant asséner le même jour que nous avons une nouvelle collectivité et que nous reconnaissons que nous sommes une nation disent : hop cé boug la a dan en léta $^{19}$ (...) C'est parce que je suis un nationaliste et que je ne veux pas tuer l'idée de nation dans les masses martiniquaises que je ne peux pas voter ce terme de nation. » (PV, 2002, tome $1: 106 / 107)$.

Ce décalage des conceptions et représentations est-il dû à l'acculturation de la population martiniquaise, comme le prétend son collègue et co-militant Jean-Claude Duverger ?

«(...) prenons notre temps pour expliquer aux gens victimes de l'acculturation ${ }^{20}$ ce que c'est que la nation. » (PV, 2002, tome 1 : 100).

«(...) Aujourd'hui parce que nous avons subi une acculturation, parce qu'on ne nous a jamais appris notre histoire, nous avons un peuple qui confond la nation et l'Etat (...) $\gg(\mathrm{PV}, 2002$, tome $1: 111)$.

Ces propos méritent tout d'abord une brève clarification quant au terme «acculturation». Il me semble que son emploi dans ce contexte est

${ }^{18}$ C'est nous qui soulignons.

${ }^{19}$ «Ces messieurs ont maintenant créé un Etat ( (traduction non littérale).

${ }^{20} \mathrm{C}$ 'est nous qui soulignons. 
imprécis voire incorrect. Car si on se réfère à la définition établie par l'anthropologie culturelle nord-américaine, créatrice de ce concept, l'acculturation serait "l'ensemble des phénomènes qui résultent du contact continu et direct entre des groupes d'individus de cultures différentes, avec des changements subséquents dans les types (patterns) culturels originaux de l'un ou des deux groupes " ${ }^{21}$, un échange donc à double direction (a two-way process selon Herskovits), même si cet échange est le plus souvent inégal (Giraud, 1995: 22). Il est vrai que dans le projet de colonisation, les situations d'acculturation étaient imposées voire planifiées ce qui a pu aboutir dans certains cas à une véritable déstructuration de la culture d'origine, entraînant une déculturation selon l'expression employée par Pierre Bourdieu à propos des effets de la colonisation française en Algérie (Bloess et al., 1997: 7 f.). Cependant, ce concept subit couramment une mésinterprétation qui implique une confusion avec le concept d' «assimilation » lequel possède d'ailleurs aux Antilles une connotation très négative. «Assimilation» signifie qu'un des deux groupes en contact finit par adopter l'ensemble des traits culturels de l'autre société et abandonne sa culture d'origine (Bloess et al., op.cit.: 10). Dans le cas de la société martiniquaise, il nous semble d'ailleurs que le terme d'assimilation, au sens culturel du terme, doit être nuancé car il n'existe aujourd'hui aucun doute que les Martiniquais, malgré le processus d'assimilation, ont préservé un nombre important d'attributs d'une culture spécifiquement martiniquaise, cette culture qui depuis une vingtaine d'années fait l'objet d'une revalorisation voire d'une « patrimonialisation » (Giraud, 1999).

D'autres problèmes découlent du propos de M. Duverger. Tout d'abord, il positionne les «victimes de l'acculturation » face à ceux qui ne le seraient pas - les nationalistes - ou du moins ceux qui auraient en quelque sorte dépassé le processus de l'acculturation (puisqu'il s'agit d'un phénomène dynamique). Cela reviendrait à dire que dans la population martiniquaise, tous les individus ne seraient pas égaux vis-à-vis de l'échange culturel. Par ailleurs, dans cette distinction établie entre les nationalistes - ceux qui auraient compris le vrai sens du terme «nation martiniquaise»-et les «victimes de

\footnotetext{
${ }^{21}$ Robert Redfield, Ralph Linton, Melville J. Herskovits, 1936, « Memorandum on the Study of Acculturation », American Anthropologist, 149-152 (repris in M.J. Herskovits, 1952, Les bases de l'anthropologie culturelle, Payot, Paris), cités par Giraud (1995 : 19); Bloess / Etienne / Noreck / Roux (1997 : 7).
} 
l'acculturation », on établit déjà un fossé, un décalage entre les deux. Enfin, étant donné le caractère bilatéral du concept d'acculturation, peut-on vraiment parler de «victimes» dans ce contexte ? En réalité, ces personnes ont leur propre conception de la nation qui n'est pas sans fondements. A travers cette représentation de la nation en termes politiques et économiques, elles expriment aussi leur difficulté à concevoir la Martinique comme nation politiquement souveraine et économiquement viable, même si ce désir est souvent là et plus ou moins ouvertement exprimé. Effectivement, nous avons pu relever lors de notre enquête le sentiment largement partagé que le statut départemental a aujourd'hui atteint ses limites. Certes, nos interlocuteurs savent valoriser les apports de la départementalisation, en particulier la protection sociale, une sécurité ainsi qu'un certain confort et bien-être matériels. Mais ils en dénoncent aussi les «effets secondaires » lourds de conséquences pour la société martiniquaise, avant tout de la consommation déraisonnée et de l'assistanat généralisé d'une population qui, à la suite d'un processus de modernisation ultra-rapide a quelque peu perdu ses repères et manque de perspective et parfois aussi d'initiative.

\section{Quel projet pour la nation?}

En réalité, la résolution de la question identitaire et avec celle-ci du problème statutaire ne correspond pas aux préoccupations immédiates et prioritaires de la population laquelle, dans le cadre du Congrès, a accusé ses élus de ne pas avoir abordé les problèmes concrets auxquels la société martiniquaise se trouve confrontée : chômage, délinquance, mauvaise qualité des transports en commun, violences de toutes sortes, problèmes économiques etc. Dans le même ordre d'idées, Justin Daniel parle de l'incapacité des mouvements nationalistes ou identitaires à apporter, dans le cadre d'un projet en cohérence avec l'idéal indépendantiste ou autonomiste "des réponses crédibles aux interrogations diverses et légitimes d'une population sans doute sensible au fait identitaire, mais qu'une réalité complexe oblige à beaucoup de discernement dans le choix de ses options politiques »(Trésor, 1999, cité par Daniel, 2002: 598). Certains de mes interlocuteurs parlent même d'un débat complètement inutile, car fondé sur des concepts et des termes qui n'apportent aucun progrès :

«(...) est-ce que cet intitulé «peuple martiniquais » apportera au Martiniquais son épanouissement? Je crois que si le Martiniquais lui-même ne s'engage pas à se faire valoir, à s'auto-identifier par ses actions, par son comportement, par sa créativité, ce n'est pas une 
inscription manuscrite sur un papier, qui fera du Martiniquais (...) que ce soit peuple ou nation ou quoi que ce soit, ce qui m'intéresse c'est de voir le Martiniquais réagir et se responsabiliser (...) » (Un chef d'entreprise de 43 ans, Le Marin).

«(...) c'est encore un acte de mimétisme. Si je suis suffisamment fort dans ma personne, bien dans ma peau, je n'ai pas besoin qu'on me reconnaisse en tant que tel parce que moi je n'aurai pas besoin de l'affirmer. (...) l'homme n'a pas besoin de se présenter: quand je vais en France, je n'ai pas besoin de dire que je suis noir! Tout le monde le voit, ça ne sert à rien de crier: je suis noir. (...) La reconnaissance du peuple martiniquais... on parle de tant de choses qui n'ont pas d'intérêt majeur et immédiat, ça c'est dans le verbe, c'est dans le discours, dans les masturbations qu'ils ont dans les sièges dans lesquels ils sont payés là, mais ça n'amène le pays à rien ! (...) » (Un autre chef d'entreprise de 52 ans, Le Marin).

Pour résumer, " c'est le projet nationaliste qui fait exister la nation » (Giraud, 2000: 92), et comme il n'y a pas de véritable projet de société derrière, la nation manque de substance. Le discours performatif (terme emprunté à Bourdieu) employé par les nationalistes dans l'objectif de «faire advenir ce qu'il énonce dans l'acte même de l'énoncer» (Bourdieu, 2001 : 286) manque alors d'efficacité ${ }^{22}$. Pour suivre l'argumentation de Bourdieu, ce manque d'efficacité pourrait être dû au fait que les nationalistes, donc ceux qui emploient ce discours, n'auraient pas réussi jusqu'à présent à

\footnotetext{
${ }^{22}$ «L'acte de magie sociale qui consiste à tenter de produire à l'existence la chose nommée peut réussir si celui qui l'accomplit est capable de faire reconnaître à sa parole le pouvoir qu'elle s'arroge par une usurpation provisoire ou définitive, celui d'imposer une nouvelle vision et une nouvelle division du monde social. (...). Mais l'effet de connaissance qu'exerce le fait de l'objectivation dans le discours ne dépend pas seulement de la reconnaissance accordée à celui qui le tient; il dépend aussi du degré auquel ce discours qui annonce au groupe son identité est fondé dans l'objectivité du groupe auquel il s'adresse, c'est-à-dire dans la reconnaissance et la croyance que lui accordent les membres de ce groupe autant que dans les propriétés économiques ou culturelles qu'ils ont en commun, puisque c'est en fonction seulement d'un principe déterminé de pertinence que peut apparaître la relation entre ces propriétés. Le pouvoir sur le groupe qu'il s'agit de porter à l'existence en tant que groupe est inséparablement un pouvoir de faire le groupe en lui imposant des principes de vision et de division communs, donc une vision unique de son identité et une vision identique de son unité. » (Bourdieu, op.cit. : 286).
} 
représenter une autorité et un pouvoir de définition reconnus comme légitimes par la population martiniquaise. Mais cette autorité peut-elle s'ériger à partir d'un discours, donc un acte d'énonciation seul, sans actions concrètes démontrant son bien-fondé ?

\section{$L a$ « dignité » comme projet politique}

Le débat autour de la reconnaissance de la nation martiniquaise et du peuple martiniquais nous montre que le projet nationaliste en Martinique - si l'on veut parler en terme de projet - est essentiellement un projet de "dignité » bâti autour du thème identitaire. On remarque par ailleurs que le terme même de «dignité » constitue un thème récurrent dans des déclarations et discours politiques. En réaction à l'humiliation coloniale que les Martiniquais ont subi dans le passé et continuent à subir par la présence et domination $\mathrm{du}$ «blanc », les nationalistes recherchent une reconnaissance avant tout extérieure mais aussi intérieure sans pour autant pouvoir et/ou vouloir aller au bout de cette reconnaissance qui signifierait l'accès à l'indépendance nationale. Mais qu'est-ce que la dignité ? Existe-il une dignité objectivement définissable et reconnue universellement en ces termes ? La dignité d'une société peut effectivement être liée au fait d'avoir une identité propre et une culture particulière, reconnues en tant que telles à l'intérieur comme à l'extérieur de cette société. On peut aller plus loin en prétendant qu'une société ne peut acquérir son entière dignité que lorsqu'elle est devenue politiquement souveraine et indépendante. D'un autre côté, une société peut-elle vivre dans la dignité si elle est certes reconnue comme nation mais économiquement dépendante d'un tourisme «bas de gamme » destructeur de son environnement et à un certain degré aussi de sa culture, comme c'est le cas de nombreux pays de la Caraïbe ? Peut-on parler d'une nation digne si la majorité de sa population vit dans la misère comme c'est le cas en Haïti ? Nous évoquons ces exemples car ils sont récurrents dans les discours que nous avons pu recueillir pour justifier le rejet et la peur d'une éventuelle indépendance de la Martinique. Car la population en quelque sorte «dose les portions de dignité »: certes, elle est en faveur d'une valorisation de la culture martiniquaise, elle apprécie aussi la promotion de la langue créole, cependant, il ne faut pas aller trop loin... et surtout ne pas toucher aux «droits acquis ». J'ai pu en effet constater une ambivalence dans les attitudes : d'un côté, les Martiniquais préfèrent la sécurité matérielle à la fierté d'être une nation souveraine, mais d'un autre côté, ils récusent souvent eux-mêmes cette attitude. 
Une interrogation fondamentale s'impose : un projet politique articulé autour d'un concept aussi subjectif et difficile à cerner que la dignité est-il réalisable? La politique ne se trouve-t-elle pas dans une impasse ? La dignité peut en effet devenir un moyen puissant de mobilisation politique, mais seulement à condition d'un large accord sur la forme de dignité à acquérir. Il est évident que vu la dépendance politique de la Martinique, la marge de manœuvre des élus locaux est réduite. A défaut d'un réel pouvoir de décision, ils se rabattent sur la question de l'identité et de la dignité qui sont néanmoins des notions bien trop abstraites pour une population en attente d'actions concrètes. Il faut dire que les exigences de cette dernière ne sont pas faciles à satisfaire, comme le constate très justement Justin Daniel (2002 : 599) : "La traduction politique des attentes exprimées par les populations n'est d'ailleurs pas aisée: elle doit concilier, dans un cadre juridico-institutionnel inédit, l'aspiration universaliste (le principe égalitaire républicain), la prise en compte des particularismes (l'identité culturelle) et la préservation des «droits acquis » dont certains obéissent à une logique purement instrumentale mobilisant alternativement ou simultanément le registre de l'égalitarisme républicain et celui des spécificités locales, entretenant ainsi la dépendance prétendument combattue. ».

Le hiatus entre la population et ses élus nationalistes sur la représentation de la nation est à notre avis emblématique de tout un contexte général marqué par le décalage flagrant entre la population et le milieu politique. Cette véritable défiance envers les élus apparaît comme un leitmotiv tout le long de notre enquête de terrain laquelle commence avec la $2^{\mathrm{e}}$ session du Congrès en début 2002 et finit avec la consultation populaire du 7 décembre 2003. Il nous semble impossible de régler la question statutaire tant que ce hiatus existera et tant qu'un véritable projet de société ne verra pas le jour. Ce projet pourrait être établi par les élus locaux dans un cadre de concertation comparable au Congrès. Cependant, on pourrait aussi imaginer qu'un tel projet émane directement de la population ou au moins soit conçu en étroite collaboration avec elle. Ceci supposerait néanmoins un réel potentiel d'initiative ainsi qu'un dépassement du décalage mentionné ci-dessus car comme le constatent les auteurs du Manifeste pour un projet global: "C'est le Projet qui sécrète le Statut qui lui est nécessaire, et non le contraire. ${ }^{23}$.

${ }^{23}$ Chamoiseau Patrick, Delver Gérard, Glissant Edouard, Juminer Bertène, 2000, « Manifeste pour un projet global », Antilla, $\mathrm{n}^{\circ} 867,14$ janvier 2000. 
Quant à la question de l'identité martiniquaise et plus spécialement celle de l'identité nationale, si étroitement liées au débat statutaire, même si l'on imagine que - dans le cas extrême du changement de statut - la Martinique accède un jour à l'indépendance nationale, la nation martiniquaise devra être définie, créée et recréée de l'intérieur même de la société martiniquaise. Et ce processus ne s'achèvera sans doute pas le jour de son indépendance, comme nous l'enseignent les expériences des nouvelles nations post-coloniales. Ainsi, Thomas Eriksen, en parlant de l'Ile Maurice et de Trinidad, explique que dans les deux sociétés - toutes les deux sont des démocraties parlementaires - la forme et le contenu du nationalisme ainsi que de l'appartenance nationale font objet d'un débat permanent et important (Eriksen, op.cit.: 143). C'est là que la définition de la nation comme "invention» (Gellner, 1983) mais aussi celle - si fréquemment citée - qui présentent les nations comme « communautés imaginées » (Anderson, 1983) prennent tout leur sens. Tout ceci laisse à penser que la question nationale en Martinique a encore de beaux jours devant elle, au-delà de celle plus immédiate du statut. 


\section{BIBLIOGRAPHIE ET SOURCES}

\section{Ouvrages et articles}

Anderson (Benedict), Imagined Communities. Reflections on the Origin and Spread of Nationalism, London/New York, Verso, 1983 (traduction française : L'imaginaire national, Paris, La Découverte, 1996).

Badie (Bertrand), Birnbaum (Pierre), Braud (Philippe), Hermet (Guy), Dictionnaire de la science politique et des institutions politiques, Paris, Armand Colin, 1994.

Bloess (Françoise), Etienne (Jean), Noreck (Jean-Pierre), Roux (Jean-Pierre), Dictionnaire de sociologie. Les notions, les mécanismes, les auteurs, $2^{\mathrm{e}}$ édition, Paris, Hatier, 1997.

Bourdieu (Pierre), Langage et pouvoir symbolique, Paris, Fayard, 2001.

Daniel (Justin), «Régionalisation et décentralisation à la Martinique : ajustements à la marge et intégration politique », Les Temps Modernes, maijuin, 1989, p. 223-242.

Daniel (Justin), «L'espace politique aux Antilles françaises », Ethnologie française, XXXII, 2002, 4, p. 589-600.

Darsières (Camille), Des origines de la nation martiniquaise, Pointe-à-Pitre, Désormeaux, 1974.

Delannoi (Gil) et Taguieff (Pierre-André) (sous la direction de), Théories $d u$ nationalisme, Paris, Kimé, 1991.

Elwert (Georg), «Ethnizität und Nation », in Joas (Hans) (sous la direction de), Lehrbuch der Soziologie, Frankfurt/Main, Campus, 2001, p. 245-263.

Eriksen (Thomas Hylland), Us and Them in Modern Societies. Ethnicity and Nationalism in Mauritius, Trinidad and Beyond, Oslo, Scandinavian University Press (Universitaetsforlaget AS), 1992.

Gellner (Ernest), Nations and Nationalism, Oxford, Blackwell, 1983 (traduction française : Nations et nationalismes, Paris, Payot, 1989. 
Giraud (Michel), «Acculturation», Pluriel Recherches. Vocabulaire historique et critique des relations inter-ethniques, $\mathrm{n}^{\circ} 3$, Paris, L'Harmattan, 1995, p. 19-25.

Giraud (Michel), «La patrimonialisation des cultures antillaises. Conditions, enjeux et effets pervers », Ethnologie française, XXIX, 1999, 3, p. 375-386.

Giraud (Michel), «Après la colonie, la nation ? Le cas des sociétés dépendantes de la Caraïbe », Pouvoirs dans la Caraïbe, Revue du C.R.P.L.C., $\mathrm{n}^{\circ} 12,2000$, p. $79-110$.

Hobsbawm (Eric), Nations and Nationalism since 1780, Cambridge University Press, 1990 (traduction française : Nations et nationalisme depuis 1780, Paris, Gallimard, 1992).

Isaacs (Harold R.), Idols of the Tribe : Group Identity and Political Change, New York, Harper and Row, 1975.

Lise (Claude), Tamaya (Michel), Les Départements d'outre-mer aujourd'hui : la voie de la responsabilité : rapport à Monsieur le Premier Ministre, France, [s.n.], Premier ministre, 1999.

Michalon (Thierry), "L'éclatement de la République intra-nationale », in Deckker P. et Faberon J.-Y., L'Etat pluriculturel et les droits aux différences, Bruxelles, éd. Bruylant, 2003, p. 185 (Actes du colloque de l'Institut de Droit de l'Outre-mer et de l'Equipe d'accueil «Identité et Oralité dans le Pacifique », tenu à Nouméa, Nouvelle-Calédonie, 3-5 juillet 2002.

Roger (Antoine), Les grandes théories du nationalisme, Paris, Armand Colin, 2001.

Rubio (Nathalie), L'avenir des départements antillais, La Documentation Française, Collection Monde européen et international, CERIC, Université d'Aix-Marseille III, 2000.

Seton-Watson (Hugh), Nations and States. An Inquiry into the Origins of Nations and the Politics of Nationalism, Boulder, Colorado, Westview Press, 1977.

Suvélor (Roland), «Eléments historiques pour une approche socioculturelle », Les Temps Modernes, n ${ }^{\circ}$ 441-442, avril-mai 1983, p. 2174-2208. 
William (Jean-Claude), «Les origines de la départementalisation », in Suvélor (Roland) (sous la direction de) Historial Antillais, Tome VI, Pointe-à-Pitre, Dajani, 1981, p. 50-61.

\section{Journaux et autres documents}

Antilla, n 867, 14 janvier 2000, p. 15-18.

Antilla, $\mathrm{n}^{\circ} 977,1^{\mathrm{er}}$ mars 2002 , p. 13-14 et 23-26.

Antilla, $\mathrm{n}^{\circ}$ 978, 8 mars 2002, p. 3-6.

Procès-verbal in extenso du $2^{e}$ Congrès des Elus Départementaux et Régionaux de la Martinique, tome 1, 20 février 2002.

\section{Entretiens}

Entretien avec Roland Suvélor du 18 février 2002.

Entretiens semi-directifs anonymes avec 28 personnes, mars-juin 2002. 


\title{
RESUME
}

Le Congrès des élus départementaux et régionaux et l'ambiguïté des revendications statutaires : «la dignité » comme projet - débat statutaire et limite de la politique locale à la Martinique.

En Martinique, le débat statutaire - étroitement lié à la question de l'identité et plus spécifiquement à celle de l'identité nationale - occupe les premières loges de la scène politique et détermine les clivages. Pourquoi le problème du statut est-il si difficile à résoudre ? Cet article tente d'apporter une réflexion à cette problématique en examinant un événement politique exceptionnel : la deuxième session du congrès des élus départementaux et régionaux, et plus particulièrement les réactions de la population et les représentations qu'elle en avait. Nous observons un décalage flagrant entre la population et ses élus, en général, et aussi plus spécialement quant à l'idée de la nation martiniquaise défendue par des élus nationalistes. Par ailleurs, les attentes de la population se situent à un niveau plus concret que les polémiques identitaires auxquelles elle a pu assister lors de ce congrès. Le projet des nationalistes semble être en premier lieu un projet de «dignité ». Mais un tel projet est-il réalisable dans le domaine de la politique?

Mots-clés : Débat statutaire, Nation, Peuple, Etat, Culture, Indépendance, Identité nationale, Identité culturelle, Représentations populaires, Dignité.

\begin{abstract}
The Congress of general and regional councillors and the ambiguity of status claims: the "dignity" as project - the status debate and the limit of local politics in Martinique.

In Martinique, the status debate, which is closely linked to the question of identity and especially to the one of national identity, occupies centre stage on the political landscape. Until now, no real solution has been found for the status problem. This paper discusses a special political event of great importance for this debate: the second session of the congress gathering the representatives of both local councils (Conseil General and Conseil Regional). In our fieldwork, we noted flagrant discrepancy between these representatives and the population, in particular with respect to the idea of the nation of Martinique promoted by the nationalist movement. Moreover the status and identity debate is far too abstract for the population in search of concrete solutions for everyday problems. The nationalists' political project seems to be first and foremost a project of "dignity". But is such a project feasible in the political field?

Key-words: Status Debate, Nation, People State, Culture, Independence, National Identity, Cultural Identity, Popular Representations, Dignity.
\end{abstract}


Ulrike ZANDER 


\section{ANNEXES}

\section{Echantillon des entretiens semi-directifs}

1. Sexe

\begin{tabular}{|c|c|c|}
\hline Femmes & Hommes & Total \\
\hline 16 & 10 & 26 \\
\hline
\end{tabular}

2. Age

\begin{tabular}{|c|c|c|c|}
\hline $\mathbf{1 5}-\mathbf{2 5}$ & $\mathbf{2 6}-\mathbf{4 0}$ & $\mathbf{4 1}-\mathbf{6 0}$ & $\mathbf{7 0 0}$ \\
\hline 0 & 13 & 9 & 4 \\
\hline
\end{tabular}

\section{Statut socio-professionnel}

\begin{tabular}{|l|l|}
\hline Agriculteurs exploitants & 1 \\
\hline $\begin{array}{l}\text { Artisans, commerçants } \\
\text { et chefs d'entreprise }\end{array}$ & 4 \\
\hline $\begin{array}{l}\text { Cadres et professions intellectuelles } \\
\text { supérieures }\end{array}$ & 2 \\
\hline $\begin{array}{l}\text { Professions intermédiaires dont : } \\
\text { Professions de l'enseignement, de la } \\
\text { santé, de la fonction publique et } \\
\text { assimilés }\end{array}$ & 7 (dont 3 enseignants) \\
\hline Employés & 7 \\
\hline Ouvriers & 2 \\
\hline $\begin{array}{l}\text { Sans activité professionnelle } \\
\text { dont chômeurs de longue durée, } \\
\text { Allocataires du RMI }\end{array}$ & 3 \\
\hline Etudiants et lycéens & 0 \\
\hline
\end{tabular}

${ }^{24}$ Revenu Minimum d'Insertion. 


\section{Milieu socio-racial}

\begin{tabular}{|l|l|}
\hline Békés & 1 \\
\hline Mulâtres & 3 \\
\hline Noirs et Indiens & 22 \\
\hline
\end{tabular}

\section{Lieu d'habitation}

\begin{tabular}{|l|l|}
\hline $\begin{array}{l}\text { Agglomération urbaine du Centre } \\
\text { (Fort-de-France - Lamentin - Schoelcher } \\
\text { - Saint-Joseph) }\end{array}$ & 3 \\
\hline Bourgs et campagnes du Sud & 20 \\
\hline Bourgs et campagnes du Nord & 3 \\
\hline
\end{tabular}

\section{Emigration}

a) Emigrés / Résidents à la Martinique

\begin{tabular}{|l|l|}
\hline Personnes ayant vécu dans l'émigration & 14 \\
\hline $\begin{array}{l}\text { Personnes ayant toujours vécu en } \\
\text { Martinique }\end{array}$ & 12 \\
\hline
\end{tabular}

b) Retours (personnes ayant vécu dans l'émigration)

\begin{tabular}{|l|l|}
\hline Rentrés à la retraite & 3 \\
\hline Rentrés pendant vie active & 8 \\
\hline $\begin{array}{l}\text { Partis juste pour les études ou le service } \\
\text { militaire }\end{array}$ & 3 \\
\hline Nés en métropole & 0 \\
\hline
\end{tabular}

